\title{
ON FINITELY GENERATED SUBGROUPS WHICH ARE OF FINITE INDEX IN GENERALIZED FREE PRODUCTS
}

\author{
A. KARRASS AND D. SOLITAR ${ }^{1}$
}

\begin{abstract}
Let $G=(A * B ; U)$ be the free product of $A$ and $B$ with the subgroup $U$ amalgamated. Various conditions are given which imply that every finitely generated subgroup $H$ containing a (nontrivial) normal subgroup of $G$ has finite index in $G$ (in such a case we say $G$ has the f.g.c.n. property). In particular, if $A$ is a noncyclic free group and $U$ is cyclic, then $G$ has the f.g.c.n. property. We use this last result to give a combinatorial proof that Fuchsian groups have the f.g.c.n. property; this was first proved by Greenberg using non-Euclidean geometry.
\end{abstract}

1. Introduction. We say that a group $G$ has the finitely generated normal (f.g.n.) property if every nontrivial f.g. normal subgroup of $G$ is of finite index (f.i.) in $G$.

$G$ has the finitely generated containing a normal (f.g.c.n.) property if every f.g. subgroup which contains a nontrivial normal subgroup of $G$ is of f.i. in $G$.

The f.g.n. property does not imply the f.g.c.n. property. For example, consider the group $P$ of all permutations on the integers. The only normal subgroups of $P$ besides the identity and $P$ itself are the subgroup consisting of all finite permutations and the subgroup consisting of all finite even permutations (see [9]); hence the only f.g. normal subgroup of $P$ is the identity subgroup, and so $P$ has the f.g.n. property. On the other hand, the subgroup $H$ generated by the transposition (12) and the infinite cycle $(\cdots,-2,-1,0,1,2, \cdots)$ contains all transpositions and hence contains the normal subgroup consisting of all finite permutations; since $H$ is countable but $P$ is not, $H$ is not of fi. in $P$; thus $P$ does not have the f.g.c.n. property.

If $G=A * B$ is a proper free product (i.e., $A \neq 1 \neq B$ ), then $G$ has the f.g.c.n. property (see [1]).

Received by the editors August 23, 1971.

AMS 1970 subject classifications. Primary 20E30, 20F05, $20 \mathrm{~F} 25$.

Key words and phrases. Generalized free products, amalgamated products, small subgroups.

1 This research was supported by grants from the National Research Council of Canada, Grant \#A5602 and Grant \#A5614. 
A generalization of this result, proved in [7], is the following: If $G=(A * B ; U)$ where $U$ is finite and $A \neq U \neq B$, and if $H$ is a f.g. subgroup containing a normal subgroup of $G$ not contained in $U$, then $H$ is of f.i. in $G$; in particular, if $U$ contains no subgroup normal in both $A$ and $B$, then $G$ has the f.g.c.n. property.

However, as soon as we try to generalize to the case where $U$ is infinite, even infinite cyclic, we encounter difficulties. For example, if

$$
G=\left\langle a, b ; a^{2}=b^{3}\right\rangle
$$

then the commutator subgroup of $G$ is f.g. but is of infinite index. Note, however, in (1) the amalgamated subgroup is of finite index in both factors. Indeed, Theorem 1 shows that if $G=(A * B ; U)$ where $U$ is of infinite index in $A$, the subgroups of $U$ are f.g., and $A$ has the f.g.n. property, then $G$ has the f.g.n. property.

To establish the more stringent f.g.c.n. property we require an additional hypothesis, namely, that $U$ be a "small subgroup" of $A$ (a precise definition of small is given in $\S 3)$. As a special case, suppose $A=(C * D ; V) \neq$ $Z_{2} * Z_{2}$ where $V$ is finite, $C \neq V \neq D$, and $V$ contains no nontrivial subgroup normal in both $C$ and $D$; then $G=(A * B ; U)$ has the f.g.c.n. property provided the subgroups of $U$ are all $f . g$.

We apply this result to prove that (hyperbolic) Fuchsian groups have the f.g.c.n. property. This was first proved in [3] using geometrical methods and later proved in [4] using topological techniques. Both of these papers actually establish the "f.g. containing a subnormal property". By an observation in [2], the method employed here can be used to establish this more general property.

Theorem 2 may be of independent interest. It implies a generalization of the well-known result that a proper free product cannot be a proper direct product: If $G$ is a proper free product, and $G=H K$ where $H, K$ are f.g. subgroups of $G$, then $H$ or $K$ is of f.i. in $G$.

For other conditions which yield the f.g.c.n. property for amalgamated products and also yield the f.g. intersection property see [2].

2. The f.g.n. property.

THEOREM 1. Let $G=(A * B ; U)$ where $U$ is of infinite index in $A, A$ has the f.g.n. property, and the subgroups of $U$ are f.g. Then $G$ has the f.g.n. property.

Proof. Let $H$ be a f.g. normal subgroup of $G$. Since $U$ is of infinite index in $A, H$ is not contained in $U$. Therefore by Theorem 10 of [7], $H U$ is of f.i. in $G$. Hence (using the notation of [7]) the collection $\left\{D_{\alpha} E_{u}\right\}$ of 
$u$-double coset representatives for $G \bmod (H, U)$ is finite. Now for a fixed $D_{\alpha}$, the associated collection $\left\{E_{u}\right\}$ is a double coset representative system for $A \bmod \left(A \cap D_{\alpha}^{-1} H D_{\alpha}, U\right)$; since $U$ is not of finite index in $A$, $A \cap H=A \cap D_{\alpha}^{-1} H D_{\alpha} \neq 1$ and is a normal subgroup of $A$. Moreover, since every subgroup of $U$ is f.g., it follows as in the proof of Theorem 8 of [7] that $A \cap H$ is f.g., and so $A \cap H$ is of f.i. in $A$. But $H A$ has f.i. in $G$; consequently, $H$ is of f.i. in $G$.

To show that the f.g.n. hypothesis on $A$ is necessary (even if $B \neq U$ ), let $A=\left\langle x, y ; x^{2}=y^{3}\right\rangle, B=\langle b\rangle, U=g p\left(y^{2}\right)=g p\left(b^{3}\right)$. Then the commutator subgroup $G^{\prime}$ of $G=(A * B ; U)$ is f.g. ( $G^{\prime} Z$ has f.i. in $G$, where $Z$ is the center of $G$ ) but $G^{\prime}$ is of infinite index in $G$.

3. Small subgroups. Let $U$ be a subgroup of a group $A$. We say that $U$ is a small subgroup of $A$ if whenever $(H, U)$ has finite double coset index in $A$ for a f.g. subgroup $H$, then $H$ itself has f.i. in $A$.

If $A$ is infinite and $U$ is a small subgroup of $A$, then $U$ must be of infinite index in $A$. For otherwise, $(1, U)$ has $\mathrm{f}$.i. in $A$ although 1 has infinite index in $A$.

Any finite subgroup is small. If $U$ is small in $A$, then $U$ is small in any finite extension of $A$.

It is also clear that any subgroup of a small subgroup of $A$ is small in $A$.

Let $A=C * D, C \neq 1 \neq D$, be f.g.; then any nontrivial normal subgroup is large (i.e., not small) in $A$. For, suppose $C \neq Z_{2}$; it is easy to show that $N$ contains an element $n$ whose reduced form begins in a $D$-syllable and ends in a $C$-syllable. Let $H=\operatorname{gp}\left(n^{-1} C n, D\right)$. Clearly $[G:(H, N)]=$ $[G: H N]=1$. On the other hand, if we choose $1 \neq d \in D$ and $1 \neq c \in C$ so that $c$ is different from the last syllable of $n$, then no positive power of $d c$ is in $H$; hence [ $G: H]$ is infinite and $N$ is large. If $C=D=Z_{2}$, then $N$ has finite index in $A$ and $N$ is therefore large.

THEOREM 2. Let $A=(C * D ; V)$ where $V$ is finite and $C \neq V \neq D$. Then any f.g. subgroup of infinite index in $A$ is small.

Proof. Let

$$
a_{1} \cdots a_{r}
$$

be a reduced form of an element of $a$, i.e., $a_{i}$ is in one of the factors $C, D$ but $a_{i}, a_{i+1}$ are not both in the same factor. A segment of (2) is a reduced form $a_{j} a_{j+1} \cdots a_{k}, 1 \leqq j, k \leqq r$. If $g_{1} \cdots g_{r}$ is another reduced form of the element defined by (2) in $A$, then $g_{j} \cdots g_{k} \in V a_{j} \cdots a_{k} V$. For, $g_{i} \cdots g_{r} a_{r}^{-1} \cdots a_{i}^{-1} \in V$ since $g_{1} \cdots g_{r} a_{r}^{-1} \cdots a_{1}^{-1}=1$; hence

$$
g_{j} \cdots g_{k}=\left(g_{j} \cdots g_{r} a_{r}^{-1} \cdots a_{j}^{-1}\right) a_{j} \cdots a_{k}\left(a_{k+1} \cdots a_{r} g_{r}^{-1} \cdots g_{k+1}^{-1}\right),
$$

which is in $V a_{j} \cdots a_{k} V$. 
We call two elements $a, a^{\prime}$ of $A$ equivalent (denoted $a \sim a^{\prime}$ ) if $a^{\prime} \in V a V$. By the preceding remark, if an element is equivalent to a segment of one reduced form of $a$, then it is equivalent to the corresponding segment of any other reduced form of $a$ (and in fact to the corresponding segment of an element equivalent to $a$ ).

Suppose $w$ is equivalent to a segment of the product $x y z$ but $w$ is not equivalent to a segment of $x, y, z, x y$ or $y z$. Then $w \sim x_{1} y z_{1}$ where $x_{1}$ is a terminal segment of a given reduced form of $x$ and $z_{1}$ is an initial segment of a given reduced form of $z$. We prove this by induction on the sum $|x|+|z|$ of the syllable lengths of the given reduced forms of $x$ and $z$. Clearly, neither $x$ nor $z$ is in $V$. Suppose first that $|x|=|z|=1$; then it is easy to show by examining reduced forms of $y$ and $x y z$ that $w \sim x y z$. Suppose then $|x|+|z|>2$, and let $x=a x^{\prime}, z=z^{\prime} b$ where $a$ and $b$ are the first and last syllables respectively of the given reduced forms of $x$ and $z$. Then $w$ is equivalent to a segment of $a\left(x^{\prime} y z^{\prime}\right) b$. If $w$ is not equivalent to a segment of $x^{\prime} y z^{\prime},\left(a x^{\prime}\right) y z^{\prime}$, or $x^{\prime} y\left(z^{\prime} b\right)$, then by the preceding case, $w \sim x y z$; on the other hand, if $w$ is equivalent to a segment of $x^{\prime} y z^{\prime},\left(a x^{\prime}\right) y z^{\prime}$, or $x^{\prime} y\left(z^{\prime} b\right)$, then by inductive hypotheses, $w \sim x_{1} y z_{1}$, where $x_{1}$ and $z_{1}$ are as asserted.

Let $H$ be any f.g. subgroup of infinite index in $A$. We show that there exists an element $w$ of $A$ such that $w$ is not equivalent to any segment of any element of $H$. Our proof is based on the proof for the corresponding result in free groups given by G. Higman in [5]. Suppose every element $w$ of $A$ is equivalent to some segment of some element of $H$. Let $h_{1}, \cdots, h_{s}$ be given reduced forms for a finite set of generators of $H$. Since $V$ is finite, there are only finitely many elements equivalent to a segment of $h_{i}^{\varepsilon_{i}}$, $\varepsilon_{i}= \pm 1$. Suppose now $w$ is equivalent to a segment of $h_{i_{1}}^{v_{1}} \cdots h_{i_{n}}^{v_{n}}, v_{i}= \pm 1$, where $n$ is minimal. If $n>1$, then $w$ is equivalent to a segment of $x y z$ where $x=h_{i_{1}}^{v_{1}}, y=h_{i_{2}}^{v_{2}} \cdots h_{i_{n-1}}^{v_{n-1}}, z=h_{i_{n}}^{v_{n}}$; hence by the above, $w$ is equivalent to $x_{1} y z_{1}$ where $x_{1}$ and $z_{1}$ are segments of $h_{i}^{\varepsilon_{i}}$. Since $y \in H$, it follows that $A$ is the union of finitely many sets of the form $a_{i} H b_{i}=\left(a_{i} H a_{i}^{-1}\right) a_{i} b_{i}$, i.e., $A$ is the union of finitely many cosets of finitely many conjugates of $H$. But Lemma 4.1 in [8] then implies that $H$ must be of f.i. in $A$, contrary to hypothesis.

To prove Theorem 2, let $U$ be a f.g. subgroup of infinite index in $A$. We show $(H, U)$ has infinite double coset index in $A$. For, suppose $A$ is the union $H g_{i} U, i=1, \cdots, n$. Let $w_{1}, w_{2}$ be reduced forms not equivalent to any segments of any elements of $H, U$ respectively. Let $p$ be a reduced form such that $w_{1} p w_{2}$ is reduced and $|p|>\max \left|g_{i}\right|$. For some $j, g_{j}=h^{-1} w_{1} p w_{2} u^{-1}$, with $h \in H, u \in U$. Since $w_{1}$ is not equivalent to an initial segment of $h$, the last syllable of $h^{-1} w_{1}$ comes from the same factor as the last syllable of $w_{1}$; similarly, the first syllable of $w_{2} u^{-1}$ comes from the same 
factor as the first syllable of $w_{2}$. Hence $\left|g_{j}\right| \geqq|p|$, contrary to the choice of $p$.

Thus $U$ is a small subgroup of $A$ if $U$ is f.g. and of infinite index in $A$.

Corollary. Let $A=C * D, C \neq 1 \neq D$. If $H$ is a f.g. subgroup of $A$ and contains a nontrivial normal subgroup $N$ of $A$ then $H$ is of f.i. in $A$.

Proof. We first show that $C$ and $D$ are f.g. For suppose say $C$ is infinitely generated. Since $H$ is f.g., there exist f.g. subgroups $C^{\prime}, D^{\prime}$ of $C, D$ respectively, such that $H<C^{\prime} * D^{\prime}$. Hence, $N<C^{\prime} * D^{\prime}$. If $c \in C-C^{\prime}$, then $c n c^{-1} \notin C^{\prime} * D^{\prime}$ if $n \neq 1$. Thus $C, D$ are f.g.

But if $H$ has infinite index in $A, H$ is small and therefore $N$ is small; but $N$ is large by the remark immediately preceding Theorem 2.

This corollary was proved in [1] by a different method.

Corollary. Suppose $A=(C * D ; V), V$ finite, $C \neq V \neq D$. If $A=H K$ where $H, K$ are f.g. subgroups of $A$, then $H$ or $K$ is of f.i. in $A$. In particular, if $A$ is the direct product $H \times K$, then $C, D, V$ can be written in the form $C=C^{\prime} \times Y, D=D^{\prime} \times Y, V=V^{\prime} \times Y$ where $Y$ is either $H$ or $K$.

Proof. Since the double coset index of $(H, K)$ is 1 , the first assertion holds. If $A=H \times K$, then $H$ or $K$ must be finite, say $K$. Since $K$ is finite and normal, $K$ must be in $C$ or $D$, and hence within $V$. Take $C^{\prime}=C \cap H$, $D^{\prime}=D \cap H, V^{\prime}=V \cap H, Y=K$.

\section{The f.g.c.n. property.}

THEOREM 3. Let $G=(A * B ; U)$ where $U$ is a small subgroup of $A$, $A \neq U \neq B$ and the subgroups of $U$ are all f.g. If $H$ is a f.g. subgroup of $G$ and contains a normal subgroup $N, N \nless U$, then $H$ is off.i. in $G$. In particular, if $U$ contains no nontrivial subgroup normal in both $A$ and $B$, then $G$ has the f.g.c.n. property.

Proof. By Theorem 10 of [7], $(H, U)$ has finite double coset index in $G$. Hence as in the proof of Theorem 1, for a fixed $\alpha$-double coset representative $D_{\alpha}$, the double coset index of $\left(A \cap D_{\alpha}^{-1} H D_{\alpha}, U\right)$ is finite in $A$ and $A \cap D_{\alpha}^{-1} H D_{\alpha}$ is f.g. Since $U$ is small in $A, A \cap D_{\alpha}^{-1} H D_{\alpha}$ is of f.i. in $A$. Moreover, since $(H, A)$ is of f.i. in $G$, it follows that $H$ is of f.i. in $G$.

As R. Burns [2] has observed, Theorem 10 of [7] holds if $N$ is assumed merely to be subnormal in $G$; consequently, Theorem 3 holds with "normal" replaced by "subnormal".

Corollary. Suppose $A=(C * D ; V) \neq Z_{2} * Z_{2}, V$ finite, $C \neq V \neq D$, and $V$ contains no nontrivial subgroup normal in both $C$ and $D$. Then 
$G=(A * B ; U)$ has the f.g.c.n. property provided the subgroups of $U$ are f.g.

Proof. Clearly $V$ is not of index two in $C$ and $D$. Hence $A$ contains a free subgroup of rank two. Since the subgroups of $U$ are all f.g., $U$ cannot be of finite index in $A$. Therefore $U$ is small in $A$ and cannot contain a nontrivial normal subgroup of $A$.

5. The f.g.c.n. property for Fuchsian groups. A (hyperbolic) Fuchsian group (or equivalently, a discrete group of orientation-preserving motions of the hyperbolic plane) $G$ is either a free product of cyclic groups or has a presentation

$$
\begin{aligned}
G=\left\langle a_{1}, b_{1}, \cdots, a_{n}, b_{n}, c_{1}, \cdots, c_{t} ;\right. \\
\left.c_{1}^{\gamma_{1}}, \cdots, c_{t}^{\gamma_{t}}, c_{1}^{-1} \cdots c_{t}^{-1}\left[a_{1}, b_{1}\right] \cdots\left[a_{n}, b_{n}\right]\right\rangle
\end{aligned}
$$

with $n, t \geqq 0, \gamma_{i}>1$, and $\mu(G)>0$ where

$$
\mu(G)=2 n-2+\left(1-\gamma_{1}^{-1}\right)+\left(1-\gamma_{2}^{-1}\right)+\cdots+\left(1-\gamma_{t}^{-1}\right) .
$$

Greenberg [3] has shown geometrically that these discrete hyperbolic groups have the f.g.c.n. property. We sketch here a combinatorial proof of this result.

If $G$ is a free product of cyclic groups, then $G$ has the f.g.c.n. property. Suppose $G$ is as in (3). Then $G$ is known to be a finite extension of a group of the form (3) with $t=0$ and $n \geqq 2$; also $G$ cannot contain a finite nontrivial normal subgroup (for combinatorial arguments see the last part of [6]). Now such a subgroup has the f.g.c.n. property, since it can be written in the form $(A * B ; U)$ with $A=\left\langle a_{1}, b_{1}\right\rangle, \quad B=\left\langle a_{2}, b_{2}, \cdots, a_{n}, b_{n}\right\rangle, \quad U=$ $\operatorname{gp}\left(\left[b_{1}, a_{1}\right]\right)=\operatorname{gp}\left(\left[a_{2}, b_{2}\right] \cdots\left[a_{n}, b_{n}\right]\right)$, which satisfies the conditions of the corollary to Theorem 3 . Consequently $G$ itself has the f.g.c.n. property.

Similarly, one can show that $G$ has the "f.g. containing a subnormal" property.

\section{REFERENCES}

1. B. Baumslag, Intersections of finitely generated subgroups in free products, J. London Math. Soc. 41 (1966), 673-679. MR 33 \#7396.

2. R. Burns, On the finitely generated subgroups of an amalgamated product of two groups, Trans. Amer. Math. Soc. 169 (1972), 293-306.

3. L. Greenberg, Discrete groups of motions, Canad. J. Math. 12 (1960), 414-426. MR 22 \#5932.

4. H. B. Griffiths, A covering-space approach to theorems of Greenberg in Fuchsian, Kleinian, and other groups, Comm. Pure Appl. Math. 20 (1967), 365-399; Correction, ibid. 21 (1968), 521-522. MR 35 \#2282; MR 38 \#1671.

5. G. Higman, Some problems and results in the theory of groups. II, Notes of a Mini-Conference, Oxford, 12th and 13th August 1966, pp. 15-16. 
6. A. H. M. Hoare, A. Karrass and D. Solitar, Subgroups of infinite index in Fuchsian groups, Math. Z. 125 (1972), 59-69.

7. A. Karrass and D. Solitar, The subgroups of a free product of two groups with an amalgamated subgroup, Trans. Amer. Math. Soc. 150 (1970), 227-255. MR 41 \#5499. 8. B. H. Neumann, Groups covered by permutable subsets, J. London Math. Soc. 29 (1954), 236-248. MR 15, 931.

9. J. Schreier and S. Ulam, Über die Permutationsgruppe der natürlichen Zahlenfolge, Studia Math. 4 (1933), 134-141.

Department of Mathematics, York University, Downsview, Ontario, Canada 